\title{
Brote grave en un paciente con enfermedad de Crohn y coinfección por SARS-CoV-2: abordaje clínico y tratamiento
}

\section{Severe flare-up in a patient with Crohn's disease and coinfection by SARS- CoV-2: Clinical approach and treatment}

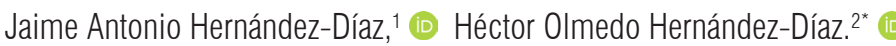

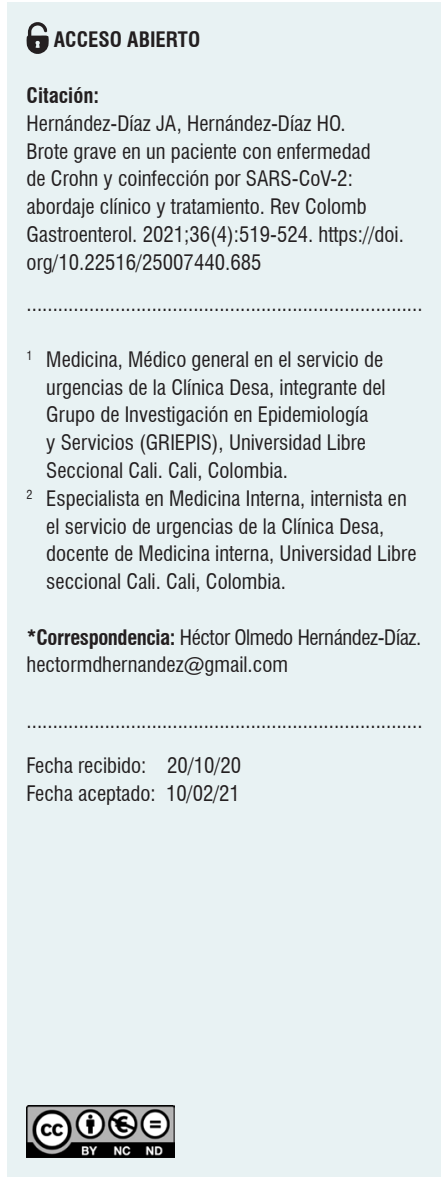

\begin{abstract}
Resumen
La enfermedad de Crohn se considera una enfermedad crónica inmunomediada, la cual fisiopatológicamente se caracteriza por ser una enfermedad multifactorial en la que intervienen factores ambientales y genéticos, entre otros. Los pacientes tienen riesgo de desarrollar complicaciones no solamente por la enfermedad sino también por los medicamentos utilizados para tratarla. En la actualidad, esto toma un papel relevante dada la enfermedad por el coronavirus de 2019 (COVID-19), infección que ha demostrado ser más grave en sujetos con comorbilidades, lo que aumenta la morbimortalidad. En este reporte de caso se presenta un hombre joven con antecedente de enfermedad de Crohn con síntomas y signos de obstrucción intestinal y respuesta inflamatoria sistémica, quien cursa por primera vez con un brote grave en el íleon terminal, compatible con perforación asociada a colección local e íleo adinámico secundario, y presenta una coinfección por el coronavirus del síndrome respiratorio agudo grave de tipo 2 (SARS-CoV-2). Se realiza la presentación del caso, discusión y revisión de la literatura del diagnóstico de brote grave de enfermedad de Crohn y coinfección de SARS-CoV-2, enfoque clínico y tratamiento.
\end{abstract}

\section{Palabras clave}

Enfermedad de Crohn, COVID-19, enfermedad inflamatoria intestinal, SARS-CoV-2, reporte de caso.

\section{Abstract}

Crohn's disease is considered a chronic immune-mediated disease, which physiopathologically is characterized by being a multifactorial disease in which environmental and genetic factors intervene, among others. Patients have a risk to develop complications not only related to the disease, but also because of the medication used to treat it. This risk plays a relevant role today due to the 2019 coronavirus disease, an infection that has been shown more serious in patients with comorbidities, which increases morbidity and mortality. In this study is analyzed a young man case with underlying Crohn's disease with symptoms and signs of intestinal obstruction and systemic inflammatory response. He was admitted for the first time with a severe flare-up in the terminal ileum, compatible with perforation associated with local collection and secondary adynamic ileus and presents a co-infection with the severe acute respiratory syndrome type 2 coronavirus (SARS-CoV-2). The case presentation, discussion, and review of the literature on the diagnosis of a severe flare-up of Crohn's disease and SARS-CoV-2 coinfection, clinical approach and treatment are carried out.

\section{Keywords}

Crohn's disease, COVID-19, inflammatory bowel disease, SARS-CoV-2, case report. 


\section{INTRODUCCIÓN}

La enfermedad inflamatoria intestinal (EII) comprende la colitis ulcerativa y la enfermedad de Crohn. En Colombia, la EII tiene una prevalencia estimada de 87 casos por 100000 habitantes: para la enfermedad de Crohn es de 17 por 100000 habitantes y para la colitis ulcerativa es de 113 por 100000 habitantes $^{(1)}$. Aunque la etiología exacta de la EII sigue siendo estudiada, la mayoría coincide en que existe un daño tisular dado por una respuesta inmunitaria excesiva contra la flora bacteriana luminal en personas predispuestas como resultado de la acción de diferentes factores ambientales ${ }^{(2)}$.

Estas enfermedades se caracterizan por períodos de remisión y brotes. Estos brotes son desencadenados por un sistema inmune que presenta una respuesta inadecuada crónica y, por tanto, el tratamiento farmacológico incluye una cantidad diversa de medicamentos inmunosupresores $^{(3)}$. Actualmente, el nuevo síndrome respiratorio agudo grave por el coronavirus del síndrome respiratorio agudo grave de tipo 2 (SARS-CoV-2) genera una gran preocupación en los pacientes con EII dado su estado inmunosupresor. En efecto, desde que se inició la identificación de los primeros casos de la enfermedad por el coronavirus de 2019 (COVID-19) en Wuhan, China, a finales de 2019, esta infección se ha diseminado precipitadamente a países donde la EII tiene mayor prevalencia, y genera mayor preocupación cuando existe un peor resultado clínico en pacientes con comorbilidades y coinfección de SARS$\mathrm{CoV}-2^{(4)}$. En pacientes con patologías crónicas como la EII, la aparición de COVID-19 ha generado incertidumbre en el enfoque y tratamiento, dado que no se tiene claro el efecto de esta infección con la descompensación de la patología de base, gravedad de los síntomas y manejo inmunosupresor $^{(5)}$. A continuación, se describe un caso de un paciente con enfermedad de Crohn y coinfección por SARS-CoV-2.

\section{CASO CLÍNICO}

Se trata de un hombre joven de 28 años con historia clínica de enfermedad de Crohn de 3 años de evolución no fistulizante con compromiso del íleon distal de ileítis crónica y en el ciego de colitis crónica erosiva y pseudopólipos, con clasificación de Montreal A2L3B2, se presenta a una clínica de tercer nivel de atención con cuadro de dolor abdominal generalizado, fiebre, distensión abdominal, náuseas, deposiciones líquidas sin sangre y episodios de emesis de contenido alimentario. Tiene antecedente de resección de neuroblastoma en la glándula suprarrenal en 1994 y laparotomía por apendicitis complicada en 2019. El manejo farmacológico de mesalazina $2 \mathrm{~g}$ vía oral lo tenía suspendido.
Al ser admitido, su peso de ingreso fue de $57 \mathrm{~kg}$, sus signos vitales eran significativos, con una presión arterial (PA) de 119/82 mm Hg, frecuencia cardíaca (FC) de 136 latidos por minuto $(\mathrm{lpm})$, saturación de oxígeno $\left(\mathrm{SatO}_{2}\right)$ del $98 \%$ en el aire ambiente y una temperatura de $37^{\circ} \mathrm{C}$. La proteína C-reactiva (PCR) fue de $136 \mathrm{mg} / \mathrm{L}$ (normal: $<8 \mathrm{mg} / \mathrm{L}$ ). Los hemocultivos, coprocultivo y urocultivo resultaron negativos. La tomografía axial computarizada (TAC) de abdomen total de ingreso reportó una solución de continuidad focal de la pared del íleon terminal a 3 $\mathrm{cm}$ de la válvula ileocecal, compatible con perforación, la cual se comunica con una colección relacionada con la cara del músculo psoas derecho a nivel de su tercio medio (volumen aproximado: $12 \mathrm{~cm}^{3}$ ). Hubo escasa cantidad de líquido libre en esta fosa ilíaca, moderado proceso inflamatorio de las paredes intestinales y el tejido graso locales. El íleo adinámico fue secundario. Fue valorado por las especialidades de cirugía general y coloproctología, y se consideró el manejo quirúrgico posterior al inicio del manejo antibiótico con ampicilina/sulbactam $3 \mathrm{~g}$ intravenoso y soporte nutricional, con lo que presentó mejoría del cuadro clínico.

Posteriormente, a los 7 días del ingreso presentó de nuevo un episodio de dolor abdominal, fiebre y deposiciones líquidas de moderada cantidad sin sangre con una frecuencia de 2 a 3 veces al día. Se toma una nueva tomografía axial computarizada (TAC) de abdomen total que reportó una resolución parcial de los signos de proceso inflamatorio en la región de la válvula ileocecal. No hubo evidencia de colecciones líquidas en este examen. Se decidió el escalonamiento antibiótico a piperacilina/tazobactam $4,5 \mathrm{~g}$ intravenoso.

Durante una semana el paciente presentó mejoría clínica, pero volvió a presentar alzas térmicas cuantificadas en $41,5^{\circ} \mathrm{C}$. Se decidió cultivar de nuevo con resultados negativos, perfil infeccioso negativo (virus de inmunodeficiencia humana $[\mathrm{VIH}]$, tuberculosis, hepatitis $\mathrm{B}[\mathrm{VHB}]$ y $\mathrm{C}[\mathrm{VHC}])$ y por persistencia de la fiebre se decidió tomar la prueba de reacción en cadena de la polimerasa con transcripción inversa (RT-PCR) para el SARS-CoV-2 por hisopado nasofaríngeo con resultado positivo. No presentó síntomas respiratorios en ningún momento, pero se realizó la toma de TAC de tórax, la cual se reportó dentro de los parámetros normales.

Fue valorado por la especialidad de infectología, que consideró que por tener EII activa y coinfección por SARSCoV-2 recomendaba evitar una cirugía mayor por la alta probabilidad de complicaciones. En ese orden de ideas, la especialidad de coloproctología decidió diferir el manejo quirúrgico por recomendación de la especialidad de infectología y, dado que la cirugía laparoscópica por el neumoperitoneo que se requiere se convertiría en un procedi- 
miento médico generador de aerosol, con alta probabilidad de infección nosocomial al personal de salud, se difirió el procedimiento hasta tener resultado negativo de RT-PCR para el SARS-CoV-2.

El paciente continúa en seguimiento por las especialidades de medicina interna y gastroenterología. Según el índice de actividad de enfermedad de Crohn (CDAI), se clasifica como actividad grave (466 puntos) de acuerdo con las manifestaciones clínicas de ingreso del paciente (peso: $57 \mathrm{~kg}$, peso ideal: $74 \mathrm{~kg}$, sexo: masculino, cantidad total de deposiciones en los 7 días previos: 21 , dolor abdominal: grave, estado general: terrible, masa abdominal: dudosa, hematocrito: $37 \%$, fiebre: sí). Se decidió dar una dosis inicial de ivermectina 1 gota por $\mathrm{kg}$ de peso con segunda dosis a los 10 días y se adicionó al manejo antibiótico metronidazol $500 \mathrm{mg}$ intravenoso. Se iniciaron esteroides con metilprednisolona $45 \mathrm{mg}$ intravenoso/día por 5 días (equivalencia de prednisolona a $1 \mathrm{mg} / \mathrm{kg} / \mathrm{día}$ ). Se realizó una enterorresonancia que reportó estenosis del aspecto más distal de la última asa del íleon, por engrosamiento concéntrico focal de sus paredes. No hay signos de proceso inflamatorio agudo, fistula ni colecciones líquidas.

El paciente presentó mejoría de su cuadro clínico con CDAI de 207 puntos y se decidió dar egreso al día 25 de hospitalización con manejo farmacológico de prednisolona $50 \mathrm{mg}$ oral con reducción progresiva, azatioprina (2 $\mathrm{mg} / \mathrm{kg} /$ día) $50 \mathrm{mg}$ oral cada 12 horas y mesalazina $2 \mathrm{~g}$ gránulos de liberación prolongada cada 12 horas debido al compromiso ileal y de colon derecho. Se le repitió la prueba de RT-PCR para el SARS-CoV-2 mediante hisopado nasofaríngeo dos veces y finalmente fue negativo después de 42 días de la presentación inicial. Se realizó una toma de calprotectina en materia fecal con resultado de $414 \mu \mathrm{g} / \mathrm{g}$ (normal: $<50 \mu \mathrm{g} / \mathrm{g}$ ).

El reporte de biopsia de colonoscopia resultó con ileítis aguda y crónica con erosión, colitis aguda y crónica grave ulcerada, y en el reporte de biopsia de endoscopia se observó gastritis crónica superficial y Helicobacter pylori en escasa cantidad (Figura 1). En el control ambulatorio se le realizó seguimiento con gastroenterología e infectología. Al ser trabajador de la salud, el paciente tenía el esquema de vacunación completo, por lo que las especialidades determinaron que el manejo biológico más recomendado de acuerdo con la situación clínica y social del paciente era con vedolizumab $300 \mathrm{mg}$ con esquema de inducción y mantenimiento, $y$, además, con soporte nutricional por desnutrición proteicocalórica moderada. El servicio de infectología recomendó, por inmunosupresión y riesgo real de reinfección, no trabajar en un área donde se manejen pacientes con COVID-19. El paciente refirió presentar mejoría de sus síntomas gastrointestinales, aumento de peso y mejoría en la calidad de vida, controles con PCR y volumen de sedi- mentación globular (VSG) negativos; presentó un CDAI al iniciar el manejo con caída de más de 100 puntos y actualmente es $<150$ puntos, lo que indica remisión clínica.

\section{DISCUSIÓN}

Este caso toma interés por diferentes razones; en primer lugar, por las manifestaciones específicas de COVID-19 en pacientes con EII. El cuadro clínico principal de esta infección en este grupo de pacientes es similar al de la población general, con fiebre y tos en la mayoría de ellos (77 \%-90\% y $66 \%$, respectivamente) ${ }^{(6,7)}$. En nuestro paciente nunca hubo síntomas respiratorios ni compromiso pulmonar, pero en esta infección viral se puede encontrar que hasta en el $61 \%$ de los casos se presentan síntomas gastrointestinales como anorexia $(35 \%)$, diarrea $(34 \%)$ y náuseas $(26 \%)^{(8)}$. Esta observación recomienda que en los pacientes con EII que desarrollen síntomas digestivos nuevos durante la pandemia por SARS-CoV-2, y especialmente si es diarrea, debe considerarse esta infección dentro del diagnóstico diferencial ${ }^{(9)}$.

En segundo lugar, es determinar si existe un mayor riesgo de infección en pacientes con EII. Diferentes estudios se han realizado en busca de una relación entre EII y COVID-19; se ha encontrado que el SARS-CoV-2 utiliza la enzima convertidora de angiotensina II (ECA-II) como receptor de entrada y la proteasa TMPRSS2 para ingresar a la célula ${ }^{(10-12)}$. Así mismo, se ha documentado un incremento en la expresión de este receptor en el intestino inflamado de pacientes con $\mathrm{EII}^{(13)}$. Estos hallazgos factiblemente sí muestran que existe un mayor riesgo de adquirir y de poder facilitar la entrada del SARS-CoV-2 en el organismo, pero aún se debe esperar hasta encontrar una evidencia clínica directa ${ }^{(14)}$.

En tercer lugar, el paciente tuvo un resultado positivo para infección por SARS-CoV-2 prologando. Estudios evidencian que la duración media de positividad para el virus es de 12 días aproximadamente ${ }^{(15)}$. En el caso de nuestro paciente, se debe estudiar si el uso de inmunomoduladores e inmunosupresores podría provocar una infección prologada por el SARS-CoV-2.

Finalmente, el enfoque del manejo médico y farmacológico de los pacientes con EII y COVID-19 está basado fundamentalmente en la prevención, medidas de bioseguridad y seguimiento continuo; esto por recomendaciones dadas por organizaciones internacionales como la European Crohn's and Colitis Organization (ECCO) y la International Organization For the study of Inflammatory Bowel Disease $(\text { IOIBD })^{(14-16)}$.

Así mismo, es importante determinar cuándo iniciar el manejo inmunosupresor o biológico luego de una infección por SARS-CoV-2. El paciente se presenta con una actividad grave de EII con una coinfección leve por SARS-COV 2 sin 


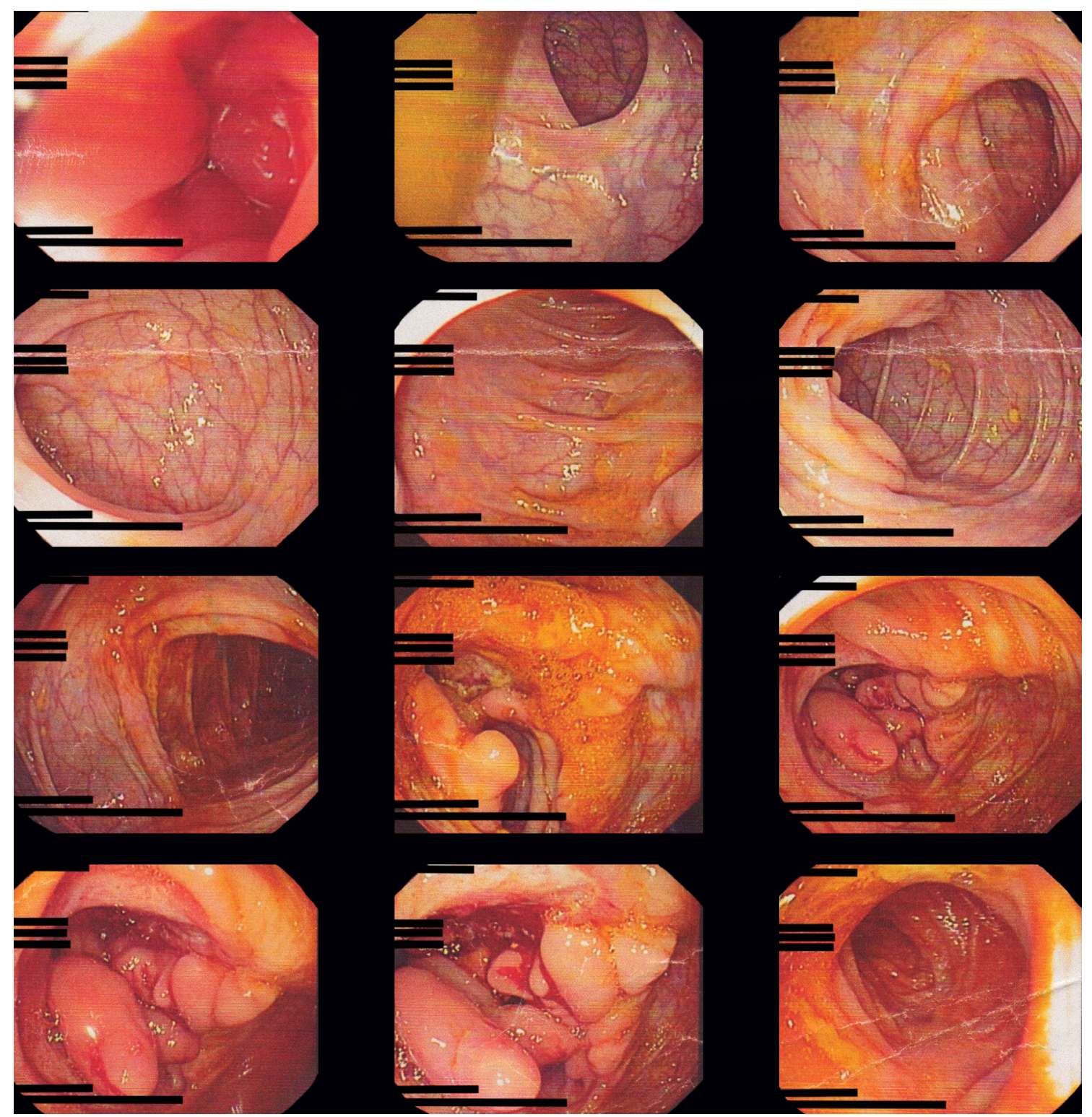

Figura 1. Hallazgos de colonoscopia total. Se exploró de forma retrógrada desde el ano hasta el íleon distal y se observó en el ciego una lesión ulcerada amplia, con bordes elevados, de aspecto mamelonado, la cual compromete la válvula ileocecal.

compromiso pulmonar, lo que permite iniciar el manejo con inmunomoduladores y esteroides sin complicaciones, aunque los diferentes estudios reportan que en casos de infección grave se debe suspender el tratamiento inmunosupresor de forma temporal o definitiva ${ }^{(16)}$. En el paciente se decidió no iniciar manejo biológico hasta obtener resultado negativo de RT-PCR para SARS-CoV-2. Los estudios recomiendan esperar al menos dos semanas para iniciar la administración de medicamentos biológicos ${ }^{(17)}$.

Actualmente existe una base de datos llamada Surveillance Epidemiology of Coronavirus Under Research
Exclusion-Inflammatory bowel disease (SECURE IBD), en la que se realiza un seguimiento de COVID-19 en pacientes con EII. Para el 8 de enero de 2021 se habían reportado 4280 casos en 64 países del mundo, liderados por Estados Unidos con un $38 \%$ de los casos, y en Colombia se han reportado 3 casos. La mortalidad observada en pacientes tratados con antiintegrinas es del $1 \%$, comparadas con 5-aminosalicílico (5-ASA), que es de $3 \%$, budesonida: $3 \%$, esteroides: $7 \%$, azatioprina: $2 \%$, metotrexato: $3 \%$, monoterapia con un antagonista del factor de necrosis tumoral alfa (anti- 
FNT- $\alpha$ ): $0 \%$, terapia combinada con inmunosupresores: $1 \%$, antiinterleucina (IL)-12/23: $1 \%$ e inhibidor JAK: $2 \%$. De acuerdo con la gravedad de la EII, los pacientes con actividad moderada/grave presentan una mortalidad del $2 \%{ }^{(18)}$. Este registro permite enfocar y comparar este caso con la población con EII y coinfección con SARS-CoV-2.
Los estudios futuros deben investigar la duración de la positividad del SARS-CoV-2 en pacientes con EII y su capacidad de transmisión; así mismo, el impacto de la inmunomodulación en el desenlace clínico de la infección y por último, buscar la relación si la infección por SARSCoV-2 sea una causa de aumento de actividad de EII.

\section{REFERENCIAS}

1. Fernández-Ávila DG, Bernal-Macías S, Parra-Izquierdo V, Rincón-Riaño DN, Gutiérrez JM, Rosselli D. Prevalencia en Colombia de la enfermedad inflamatoria intestinal y el compromiso articular asociado, según información del Sistema Integral de Información de la Protección Social. Rev Colomb Reumatol. 2020;27(1)3-8. https://doi.org/10.1016/j.rcreu.2019.10.006

2. Macdonald TT, Monteleone G. Immunity, inflammation, and allergy in the gut. Science. 2005;307(5717):1920-5. https://doi.org/10.1126/science.1106442

3. Beaugerie L, Kirchgesner J. Balancing Benefit vs Risk of Immunosuppressive Therapy for Individual Patients With Inflammatory Bowel Diseases. Clin Gastroenterol Hepatol. 2019;17(3):370-379.

https://doi.org/10.1016/j.cgh.2018.07.013

4. Zhu N, Zhang D, Wang W, Li X, Yang B, Song J, Zhao X, Huang B, Shi W, Lu R, Niu P, Zhan F, Ma X, Wang D, Xu W, Wu G, Gao GF, Tan W; China Novel Coronavirus Investigating and Research Team. A Novel Coronavirus from Patients with Pneumonia in China, 2019. N Engl J Med. 2020;382(8):727-733. https://doi.org/10.1056/NEJMoa2001017

5. Parra-Izquierdo V, Flórez-Sarmiento C, García del Risco F, Romero-Sánchez C. Síntomas gastrointestinales en la enfermedad por COVID-19 y sus implicaciones en enfermedad inflamatoria intestinal. Rev Colomb Gastroenterol. 2020;35(Supl. 1):45-55. https://doi.org/10.22516/25007440.532

6. Bezzio C, Saibeni S, Variola A, Allocca M, Massari A, Gerardi V, Casini V, Ricci C, Zingone F, Amato A, Caprioli F, Lenti MV, Viganò C, Ascolani M, Bossa F, Castiglione F, Cortelezzi C, Grossi L, Milla M, Morganti D, Pastorelli L, Ribaldone DG, Sartini A, Soriano A, Manes G, Danese S, Fantini MC, Armuzzi A, Daperno M, Fiorino G; Italian Group for the Study of Inflammatory Bowel Disease (IG-IBD). Outcomes of COVID-19 in 79 patients with IBD in Italy: an IG-IBD study. Gut. 2020;69(7):1213-1217.

https://doi.org/10.1136/gutjnl-2020-321411

7. Rodríguez-Lago I, Ramírez de la Piscina P, Elorza A, Merino O, Ortiz de Zárate J, Cabriada JL. Characteristics and Prognosis of Patients With Inflammatory Bowel Disease During the SARS-CoV-2 Pandemic in the Basque Country (Spain). Gastroenterology. 2020;159(2):781-783. https://doi.org/10.1053/j.gastro.2020.04.043
8. Redd WD, Zhou JC, Hathorn KE, McCarty TR, Bazarbashi AN, Thompson CC, Shen L, Chan WW. Prevalence and Characteristics of Gastrointestinal Symptoms in Patients With Severe Acute Respiratory Syndrome Coronavirus 2 Infection in the United States: A Multicenter Cohort Study. Gastroenterology. 2020;159(2):765-767.e2. https://doi.org/10.1053/j.gastro.2020.04.045

9. Marín-Jiménez I, Zabana Y, Rodríguez-Lago I, Marín L, Barreiro-de Acosta M, Esteve M; en representación de GETEII y GETECCU. COVID-19 and inflammatory bowel disease: questions arising from patient care and follow-up during the initial phase of the pandemic (February-April 2020). Gastroenterol Hepatol. 2020;43(7):408-413. https://doi.org/10.1016/j.gastrohep.2020.05.003

10. Xiao F, Tang M, Zheng X, Liu Y, Li X, Shan H. Evidence for Gastrointestinal Infection of SARS-CoV-2. Gastroenterology. 2020;158(6):1831-1833.e3. https://doi.org/10.1053/j.gastro.2020.02.055

11. Ahlawat S, Asha, Sharma KK. Immunological co-ordination between gut and lungs in SARS-CoV-2 infection. Virus Res. 2020;286:198103. https://doi.org/10.1016/j.virusres.2020.198103

12. Hoffmann M, Kleine-Weber H, Schroeder S, Krüger N, Herrler T, Erichsen S, Schiergens TS, Herrler G, Wu NH, Nitsche A, Müller MA, Drosten C, Pöhlmann S. SARSCoV-2 Cell Entry Depends on ACE2 and TMPRSS2 and Is Blocked by a Clinically Proven Protease Inhibitor. Cell. 2020;181(2):271-280.e8. https://doi.org/10.1016/j.cell.2020.02.052

13. Garg M, Royce SG, Tikellis C, Shallue C, Batu D, Velkoska E, Burrell LM, Patel SK, Beswick L, Jackson A, Britto K, Lukies M, Sluka P, Wardan H, Hirokawa Y, Tan CW, Faux M, Burgess AW, Hosking P, Monagle S, Thomas M, Gibson PR, Lubel J. Imbalance of the renin-angiotensin system may contribute to inflammation and fibrosis in IBD: a novel therapeutic target? Gut. 2020;69(5):841-851. https://doi.org/10.1136/gutjnl-2019-318512

14. Juliao-Baños F. Manejo de pacientes con enfermedad inflamatoria intestinal durante la pandemia de COVID-19d-19. Rev Colomb Gastroenterol. 2020;35(Supl. 1):37-44. https://doi.org/10.22516/25007440.537

15. Fiorino G, Allocca M, Furfaro F, Gilardi D, Zilli A, Radice S, Spinelli A, Danese S. Inflammatory Bowel Disease Care 
in the COVID-19 Pandemic Era: The Humanitas, Milan, Experience. J Crohns Colitis. 2020;14(9):1330-1333. https://doi.org/10.1093/ecco-jcc/jjaa058

16. Rubin DT, Abreu MT, Rai V, Siegel CA; International Organization for the Study of Inflammatory Bowel Disease. Management of Patients With Crohn's Disease and Ulcerative Colitis During the Coronavirus Disease-2019 Pandemic: Results of an International Meeting. Gastroenterology. 2020;159(1):6-13.e6. https://doi.org/10.1053/j.gastro.2020.04.002
17. Crespo J, Andrade R, Alberca de Las Parras F, Balaguer F, Barreiro-de Acosta M, Bujanda L, Gutiérrez A, Jorquera F, Iglesias-García J, Sánchez-Yagüe A, Calleja JL. Resumption of activity in gastroenterology departments. Recommendations by SEPD, AEEH, GETECCU and AEG. Rev Esp Enferm Dig. 2020;112(5):397-411. https://doi.org/10.17235/reed.2020.7141/2020

18. Kappelman MD, Brenner EJ, Colombel JF, Ungaro RC, Agrawal M. Coronavirus and IBD Reporting Database [Internet]. SECURE-IBD [consultado el 8 de enero de 2021]. Disponible en: https://covidibd.org/ 